\title{
Visualization of Food-Based Dietary Guidelines - Examples
}

\author{
Juergen S. Koenig \\ Department of Nutritional Sciences, University of Vienna, Vienna, Austria
}

\author{
Key Words \\ Visualization • Food-based dietary guidelines • \\ International guidelines • Key concepts • Translation • \\ Nutrient-based dietary guidelines
}

\begin{abstract}
Food-based dietary guidelines (FBDGs) are the primary instrument for the translation of nutrient-based guidelines into practice for the general population. The key concepts for the development of FBDGs as outlined by the report of a joint FAO/WHO consultation in 1996 are to address dietary patterns, to be practical, to be comprehensible, and to be culturally acceptable. These key concepts need to be considered particularly when planning to visualize FBDGs, since the visualization is the major mode of information transfer and the first - and quite frequently the only - contact with the guidelines. For the visualization of dietary patterns it is recommended that total diets rather than nutrients or individual foods should be addressed, although there are some examples which were successful in addressing specific foods and food groups. The design of FBDGs should be flexible for use by people with different lifestyles as well as people of different ages and with different physiological conditions. However, the visualization of FBDGs is a marketing instrument, which differs in no way from regular and commercial marketing instruments. To be successful, these instruments need to be specific for the target population. With increasing power, influence, and coverage of computer-based instruments, the individualization of FBDGs has been facilitated. New strategies have therefore been developed to provide
\end{abstract}

guidelines tailored to individual needs and preferences, as can be seen in the concept of the new food guide pyramids by the US Department of Agriculture. Whether these guidelines are successful needs to be awaited, their major problem being initiative required from the consumer rather than delivering the information appropriately and conveniently to the target population.

Copyright $\odot 2007$ S. Karger AG, Basel

\section{Introduction}

In 1992, the International Conference on Nutrition in Rome developed the Plan of Action of Nutrition [1], which emphasized the promotion of appropriate diets and healthy lifestyles as one bullet point within the nine action-oriented strategies of this plan. This strategy calls on governments 'on the basis of energy and nutrient recommendations to provide advice to the public by disseminating, through the use of mass media and other appropriate means, qualitative and/or quantitative dietary guidelines relevant for different age groups and lifestyles and appropriate for the country's population' [2]. Facing the differences in the health and nutrition situation in various countries worldwide, it is obvious that those guidelines may diverge considerably from region to region. This paper attempts to provide a short introduction into the concepts of visualization of food-based dietary guidelines (FBDGs) for selected countries and regions.

FBDGs are the primary instrument for the translation of nutrient-based guidelines into practice for the general

\section{KARGER \\ Fax +41613061234 \\ E-Mail karger@karger.ch}

www.karger.com
(C) $2007 \mathrm{~S}$. Karger AG, Basel

0250-6807/07/0518-0036\$23.50/0

Accessible online at:

www.karger.com/anm 
population. The key concepts for the development of FBDGs as outlined by the report of a joint FAO/WHO consultation in 1996 are to address dietary patterns, to be practical, to be comprehensible, and to be culturally acceptable (table 1) [3]. These key concepts need to be considered particularly when planning to visualize FBDGs, since the visualization is the major mode of information transfer and the first - and quite frequently the only contact with the guidelines.

For the visualization of dietary patterns it is recommended that total diets rather than nutrients or individual foods should be addressed, although there are some examples which were successful in addressing specific foods and food groups. The 5-a-day program in its national variations is a prominent example for not addressing total diets, but still having had some impact on nutrition behavior and awareness of the general public. It is obvious and within the nature of FBDGs that they need to reflect food patterns rather than numeric nutrient goals. This key concept however is being missed frequently. It is also important not to polarize the target population by visualizing specific diets and also to emphasize that various dietary patterns can be consistent with good health.

In respect to practicability, the recommended foods or food groups should be affordable, widely available and accessible to most people. The recommendation of increased marine fish consumption is idle for populations living far from fishing grounds and lack of logistic infrastructure. Additionally, the recognition of social, economic, agricultural, and environmental conditions affecting foods and eating patterns need to be considered. The design of FBDGs should be flexible for use by people with different lifestyles as well as people of different ages and with different physiological conditions. However, the visualization of FBDGs is a marketing instrument, which differs in no way from regular and commercial marketing instruments. To be successful, these instruments need to be specific for the target population.

Comprehensibility, immediately after first visual contact, is mandatory for visualized FBDGs, in particular to be recognized in the context of other commercials in an information overloaded society. It is obligatory that the guidelines are easily understood by the general public and the target population, respectively, and testing is essential before dissemination.

The major focus in the development of FBDGs is on the cultural acceptability of the recommendations, and this must be reflected in their visualization. The choice of foods and colors in illustrations should be culturally appropriate and sensitive to religious and other cultural
Table 1. Key principles for developing FBDGs

\section{Dietary patterns}

- Total diet, rather than nutrients or individual foods, should be addressed

- Dietary guidelines need to reflect food patterns rather than numerical nutrient goals

- Various dietary patterns can be consistent with good health Practicality

- The recommended foods or food groups should be affordable, widely available and accessible to most people

- FBDGs should recognize the social, economic, agricultural and environmental conditions affecting foods and eating patterns

- $\quad$ FBDGs should be flexible for use by people with different lifestyles as well as people of different ages and different physiological conditions

\section{Comprehensibility}

- FBDGs should be easily understood by the general public, taking into account levels of literacy. The terminology used must be simple and must refer to foods, rather than nutrients, wherever possible

- Food groups that make sense to the public should be chosen

- Visual presentation must be easily understood

- Testing of the FBDGs is essential before dissemination

\section{Cultural acceptability}

- The choice of foods and colors used in illustrations should be culturally appropriate

- The guidelines should be sensitive to religious and other cultural considerations, especially those of minority groups

- FBDGs should not recommend radical changes in current dietary practices

- Presentation should use appropriate language or dialect

- FBDGs should be positive and should encourage enjoyment of appropriate diets

considerations, especially those of minority groups. Radical changes in current dietary practices should be avoided and both wording and presentation have to be in the appropriate dialects and languages. It is a psychological standard to be positive and to encourage indulgence of appropriate diets.

\section{The Food Guide Pyramid}

Attempts to translate nutrient-based recommendations into visually comprehensible models of FBDGs have been made by various countries, frequently by adopting existing models to national and regional distinctions. The effort made by the United States Depart- 
Fig. 1. The Food Guide Pyramid from the USDA from 1992 [4].

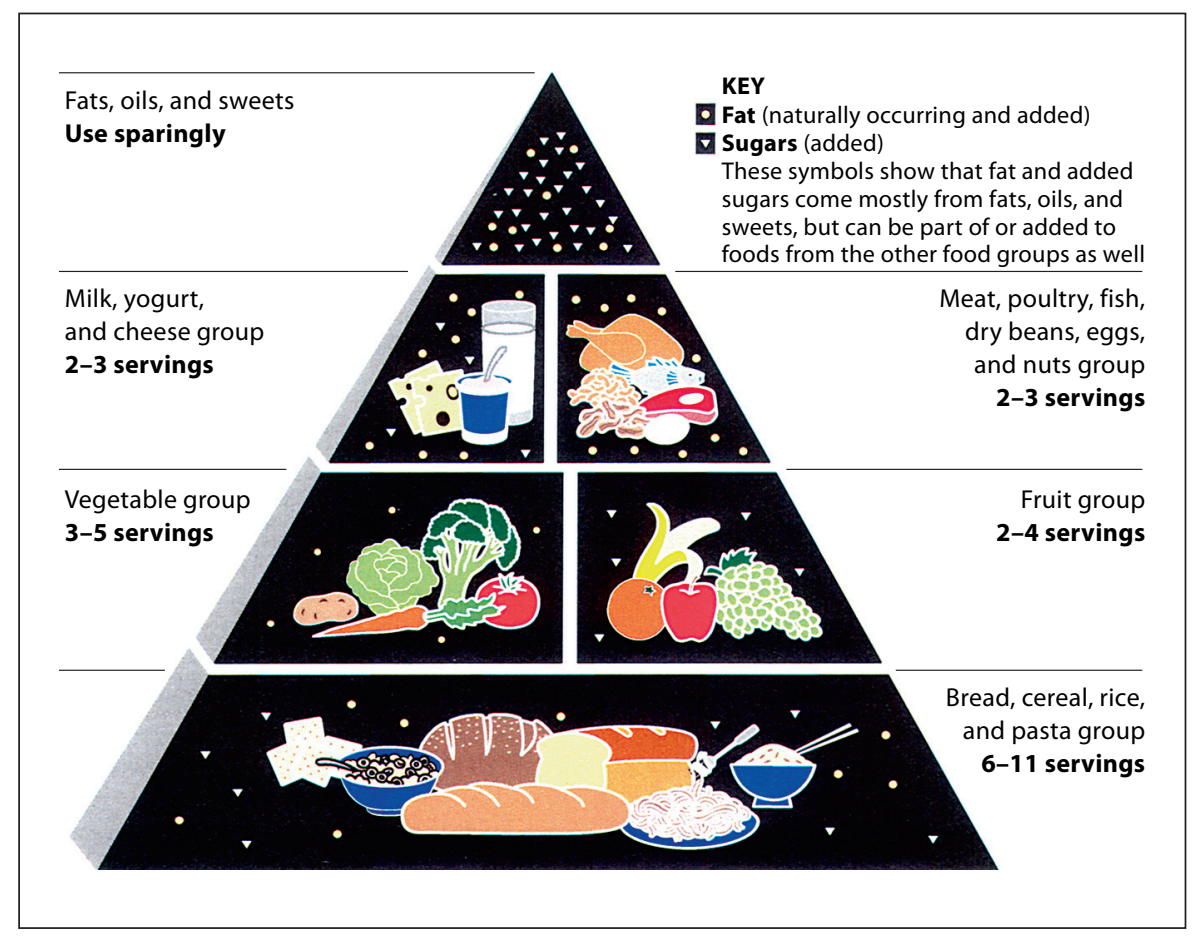

ment of Agriculture (USDA) for the development of the Food Guide Pyramid (fig. 1) [4] in the 1990s and the efforts put into harmonization of these guidelines across the North American countries placed the Food Guide Pyramid at a strong position as a role model for other countries worldwide. A WHO evaluation of FBDGs from 30 countries demonstrated that 16 of the 35 guidelines use a food pyramid [5]. The beginning of the Food Guide Pyramid's doom was the adoption by the food industry as an instrument of product-specific marketing strategies, thus leading to a tremendous loss of credibility in the public and subsequently to the development of rivalry within the sector of FBDGs. The strongest impact at the end came from the competing food pyramid developed by Walter Willett in his book 'Eat, Drink and Be Healthy' in 2001 (fig. 2) [6], in which he strongly opposed the well-established USDA Food Guide Pyramid mainly for its emphasis on carbohydrates and alleged demonization of fats.

Unfortunately, the controversial discussion of the two guidelines in the public counteracted the yield of the USDA Food Guide Pyramid gained by the unambiguous support throughout wide parts of the nutrition community and its positive impact was lost within weeks, demonstrating the susceptibility of the nutrition information market. Finally, the debate ended in the development of

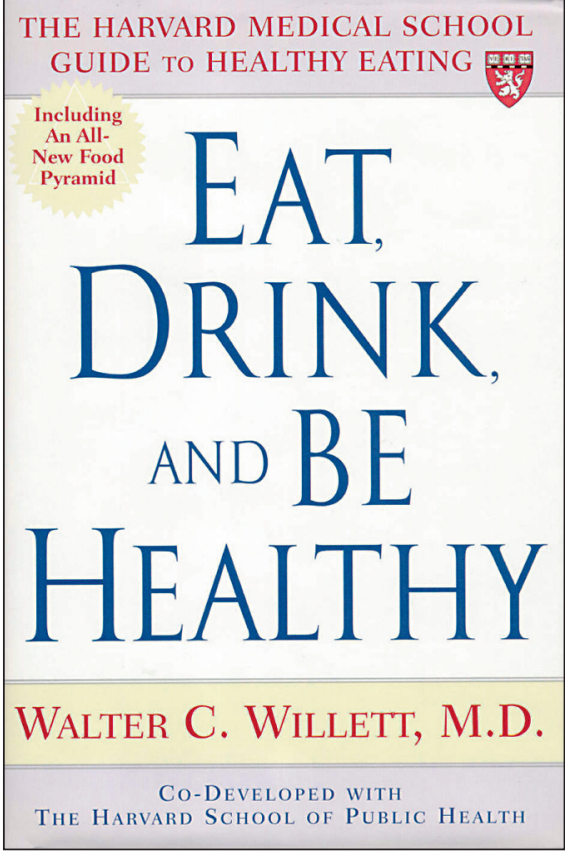

Fig. 2. 2001 book cover of 'Eat, Drink and Be Healthy' [6]. 
a modified concept of the USDA Food Guide Pyramid, the new MyPyramid.gov campaign (fig. 3) [7].

Some controversy still exists in the discussion of the appropriate design of the principle set-up of the food guide pyramid and its derivates in the various countries. Most pyramids show those foods at the base of the pyramid which predominantly supply carbohydrates (cereals, bread, rice, pasta, and the like), thus indicating this food group to be the quantitatively largest part of the diet. Fruit and vegetable recommendations are the second level of the food guide pyramid based on the amount of recommended servings. From a pure quantitative point of view, however, fruit and vegetables combined should form a larger part of the diet compared to the carbohydrate group. This has caused some confusion in the general public, again decreasing credibility of the entire concept. The situation even declines when including fluid intake into the guidelines, now resulting in fluids at the base of the pyramid. Some attempts have been made to work around these controversies, again leading to different models which in some cases have been disseminated concurrently for identical target populations.

With increasing power, influence, and coverage of computer-based instruments, the individualization of FBDGs has been facilitated. New strategies have therefore been developed to provide guidelines tailored to individual needs and preferences, as can be seen in the concept of the new food guide pyramids by the USDA. Whether these guidelines are successful needs to be awaited; their major problem being initiative required from the consumer rather than delivering the information appropriately and conveniently to the target population.

\section{National Concepts}

In view of the cultural aspects of FBDGs, it is obvious that different countries and regions have developed diverging models for the dissemination and visualization of nation- or region-specific guidelines. It is unfortunately impossible within the scope of this paper to include images of the various examples worldwide. Many of the national FBDGs have been collected by the FAO and can be assessed through their website [8].

Based on a WHO report on the global status of FBDGs, 75 countries of 126 reviewed provide a final version of guidelines for their population, 12 countries in the process of preparing guidelines, and 39 have provided no information [5]. The analysis of 64 sets of guidelines from 37 countries showed that in most cases

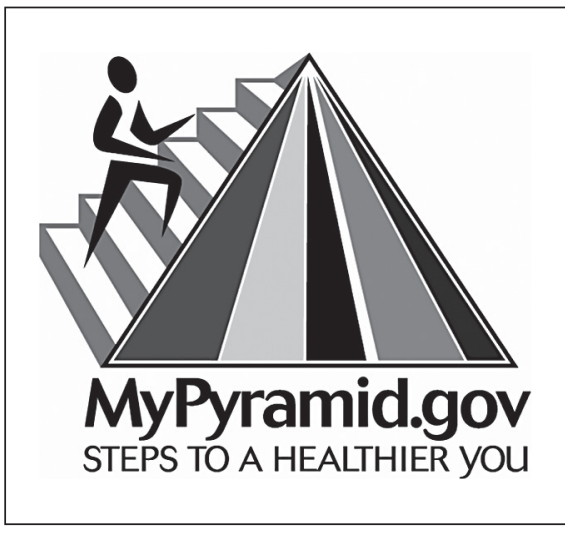

Fig. 3. MyPyramid.gov [7].

a target group is either not specified or the general population is addressed. In the same sample of guidelines, almost $80 \%$ include messages on fruit and vegetables, some $50 \%$ disseminate messages on milk and dairy products and about $30 \%$ recommendations for fish consumption. In many cases, selected nutrients are the focus of the messages, salt, fats, and sugar being the predominant topics addressed. Frequently, FBDGs also convey messages on issues not directly related to diet. Physical activity, body weight, food safety and hygiene, breastfeeding, and financial aspects are addressed in many of the sets of guidelines. Some $90 \%$ of the guidelines reviewed also include at least one social or behavioral message, e.g. enjoy your meals, make mealtime a social time, and eat a variety of foods every day. The evaluation of the messages on fruit and vegetable intake showed that 43 of the 50 messages were different to some extent, only seven delivered the same message (eat plenty of fruit and vegetables).

The approaches followed by China, Iran, Austria, Switzerland, and Germany are discussed in detail elsewhere in this issue. Therefore, some selected examples from other countries are discussed in the following.

A very similar approach to the food guide pyramid of the United States FDA has been followed by the Working Group on Food-Bases Dietary Guidelines for Thai People in the Department of Health of the Ministry of Public Health in Thailand [9]. Their concept on 'Healthy Eating for Thais' includes a Nutrition Flag, which is basically identical to the Food Guide Pyramid but has been turned upside down. This approach is appealing in respect to the typical reading direction from top to bottom in contrast to the food pyramids, and thus directing the reader's emphasis to the quantitatively most important food groups 
on first sight of the guidelines. The Thai Nutrition Flag depicts examples for the recommended food groups by selecting typical foods consumed by the Thai population which is in accordance with the FAO/WHO key concepts for the development of FBDGs. The bread, cereal, rice and pasta group from the US food guide pyramid has been converted into a group of rice-starchy food and the servings have been adopted by using rice-serving spoons as measuring unit. Again based on typical consumption patterns, the milk, yoghurt and cheese group has been reduced to a milk only group, since other milk products are of less importance in the Thai diet.

The visual concept of the Thai nutrition flag is accompanied by worded guidelines with nine rules as listed in table 2. Again in analogy to the USDA Food Guide Pyramid, the Thai FBDGs provide more detailed recommendations by visualizing examples for the recommended food groups within the Nutrition Flag and giving assistance for the conversion of household measures into the recommended servings. One plate of rice is consequently equal to three rice-serving spoons of rice, one bowl of noodles equals two rice-serving spoons, and one slice of bread, one piece of fermented noodles and one ear of corn, respectively, each represent one rice-serving spoon of those foods.

The Thailand experience in developing their FBDGs showed the importance of many of the issues addressed by $\mathrm{FAO} / \mathrm{WHO}$ in their recommendations [10]. An expert working group had been established for the development of the guidelines, which collected data on food consumption and public health-related issues prior to the development of the Thai guidelines, and data from other countries had additionally been reviewed by the working group. Similar to other countries, the working group recognized that, although appropriate data is limited, there still is no justification to abandon the process of developing national FBDGs. Institutional commitment had been identified a key issue for the process and outcome of the guidelines. Support and endorsement came from high-level governmental participation in the process of guideline development and led to the required media attention. However, some concern was expressed about the maintenance of the integrity of the messages, in particular in view of food industry using subsets of the guidelines to promote their own products. Inconsistencies had been found between the Thai Nutrition Flag and guidelines developed by private, non-governmental organizations.

Japan issued their FBDGs in a similar format as the Thai Nutrition Flag, and disseminated also a reversed food pyramid in form of a spinning top which is claimed
Table 2. FBDGs for Thailand [9]

\begin{tabular}{|c|c|}
\hline Rule 1 & $\begin{array}{l}\text { Eat a variety of foods from each of the } 5 \text { food groups } \\
\text { and maintain proper weight }\end{array}$ \\
\hline Rule 2 & $\begin{array}{l}\text { Eat an adequate amount of rice or alternative } \\
\text { carbohydrate sources }\end{array}$ \\
\hline Rule 3 & Eat plenty of vegetables and fruits regularly \\
\hline ule 4 & Eat fish, lean meats, eggs, legumes and pulses regularly \\
\hline 5 & $\begin{array}{l}\text { Drink milk in appropriate quality and quantity for } \\
\text { one's age }\end{array}$ \\
\hline 6 & Eat a diet containing appropriate amounts of fat \\
\hline & Avoid sweet and salty foods \\
\hline & Eat clean and safe foods \\
\hline & Avoid or reduce consumption of alcoholic beverages \\
\hline
\end{tabular}

to be a well-known traditional Japanese toy [11]. This has been designed to symbolize physical activity as part of the guidelines both by the dynamic motion of the spinning top itself and a pictogram of a running person on the top of the spinner. The spinning top as a symbol of balance is appealing as it may remind the target population of the importance to maintain an optimal balance in their diets. The handle of the spinning top represents fluid intake by water and tea, thus adding recommendations on fluid intake into the concept but disregarding the quantitative proportionality. Snacks, confection and beverages, which are considered non-essential treats are represented by a string attached to the side of the spinning top, symbolizing the momentum the string transfers to the spinner and the supposed momentum of these foods transferred to the Japanese diet.

Interestingly, the spinning top from Japan shows fruit at the tip of the spinning top together with milk and milk products in two separate groups. Although two servings are recommended to be consumed daily for both groups, the position at the tip implies that these food groups are of less importance than the other groups at the upper part of the spinning top, like grain dishes (5-7 servings per day) at the top and vegetable dishes (5-6 servings per day) at the second level.

The Japanese Food Guide Spinning Top is embedded into the broader concept of food education law 'Shokuiku' by the Japanese Ministry of Agriculture, Forestry and Fisheries in 2005 [11]. The basic concepts of Shokuiku are listed in table 3.

The spinning top is the visualized set of dietary guidelines developed within the same frame of food education as the spinning top itself. These guidelines are similar to dietary guidelines from other countries, demonstrating the evidence of comparable nutrition problems in the Jap- 


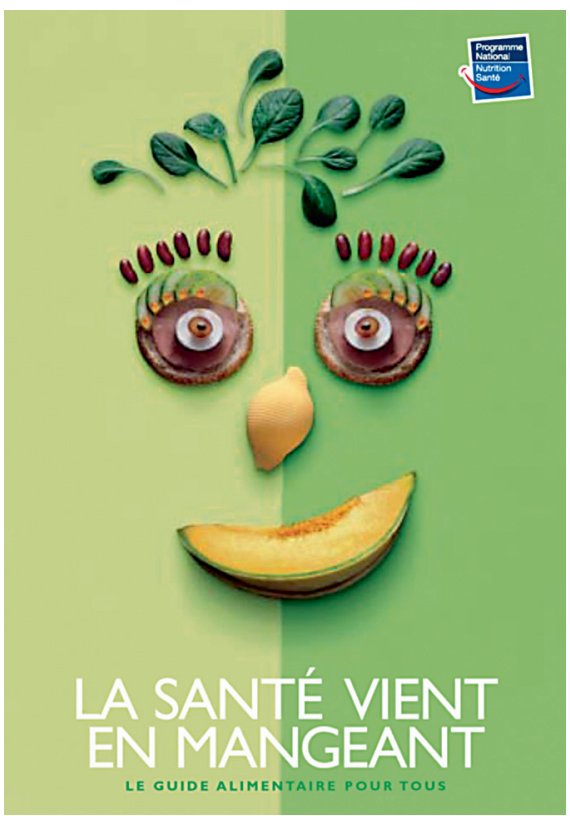

Fig. 4. French Nutrition Guide Health Comes From Nutrition [12].

anese population. The dietary guidelines for Japanese are:

- Enjoy your meals

- Establish a health rhythm by keeping regular hours for meals

- Eat well-balanced meals with staple food, as well as main and side dishes

- Eat enough grains such as rice and other cereals

- Combine vegetables, fruits, milk products, beans and fish in your diet

- Avoid too much salt and fat

- Learn your healthy body weight and balance the calories you eat with physical activity

- Take advantage of your dietary culture and local food products while incorporating new and different dishes

- Reduce leftovers and waste through proper cooking and storage methods

- Assess your daily eating.

A different approach to FBDGs has been followed by France to some extent. The French Ministry of Health and Solidarity (Ministère de la Santé et des Solidarités) issued a comprehensive 127-page booklet entitled 'Health Comes From Nutrition' (La Santé vient en mangeant) [12]. The guide attempts to cover the complete range of nutritional aspects in the different physiological, social, and econom-
Table 3. Basic concepts of the Japanese food education law 'Shokuiku'

1 Shokuiku should have the purpose of promoting people's health in body and in mind, as well as enriching human lives

2 Care should be taken to induce people to develop greater appreciation for and understanding of their diets, including the various roles played by the natural environment and the people who produce, transport and prepare food

3 Nationwide, voluntary movements for promotion of Shokuiku should be developed

4 Especially parents, educators and day-care providers should actively promote Shokuiku among children

5 Understanding of dietary issues should be reinforced by taking advantage of all available opportunities, such as at home, in schools and in the community or everywhere, to offer a variety of food-related experiences and activities

6 Awareness and appreciation of traditional Japanese food culture as well as food supply/demand situations should be promoted, and opportunities of interaction between food producers and consumers should be created, in order to revitalize rural farming and fishing regions, and to boost food self-sufficiency in Japan

7 Appropriate information on food safety should be offered, in order to help people to practice proper diets

ical situation of the French population. Although no small set of visualized guidelines has been developed, the symbolism of the French guide does include a clear line throughout the brochure by starting each chapter with recommended foods laid out in the style of the Italian painter Archimboldo and his portrait heads (fig. 4).

The French FBDGs are composed of 25 chapters with the headlines as listed in table 4 . In its introductory remarks, the guide gives as an explanation for the reason of a food guide that nutrition is one of the great pleasures of life, leading from the basic pleasure of simply not being hungry, to the higher pleasures of culinary indulgence, the joy in sharing meals with family and friends, or the gratification of not having to worry about nutrition. This is an interesting and very appealing way of putting the enjoyment of nutrition on top of the recommendations and very much represents the French attitude towards nutrition. Nonetheless, simply the length of the guide may be inappropriate for the general population and this may mislead to extract parts of the guide for specific groups of the population or specific physiological or social situations. Thus, important parts of the information provided by the guide may be missed from those concerned by various nutritional issues.

Canada developed their 'Food Guide to Healthy Eating For People Four Years and Over' in the form of a rainbow- 
Table 4. Chapters and headlines covered in the French FBDGs 'La santé vient en mangeant'

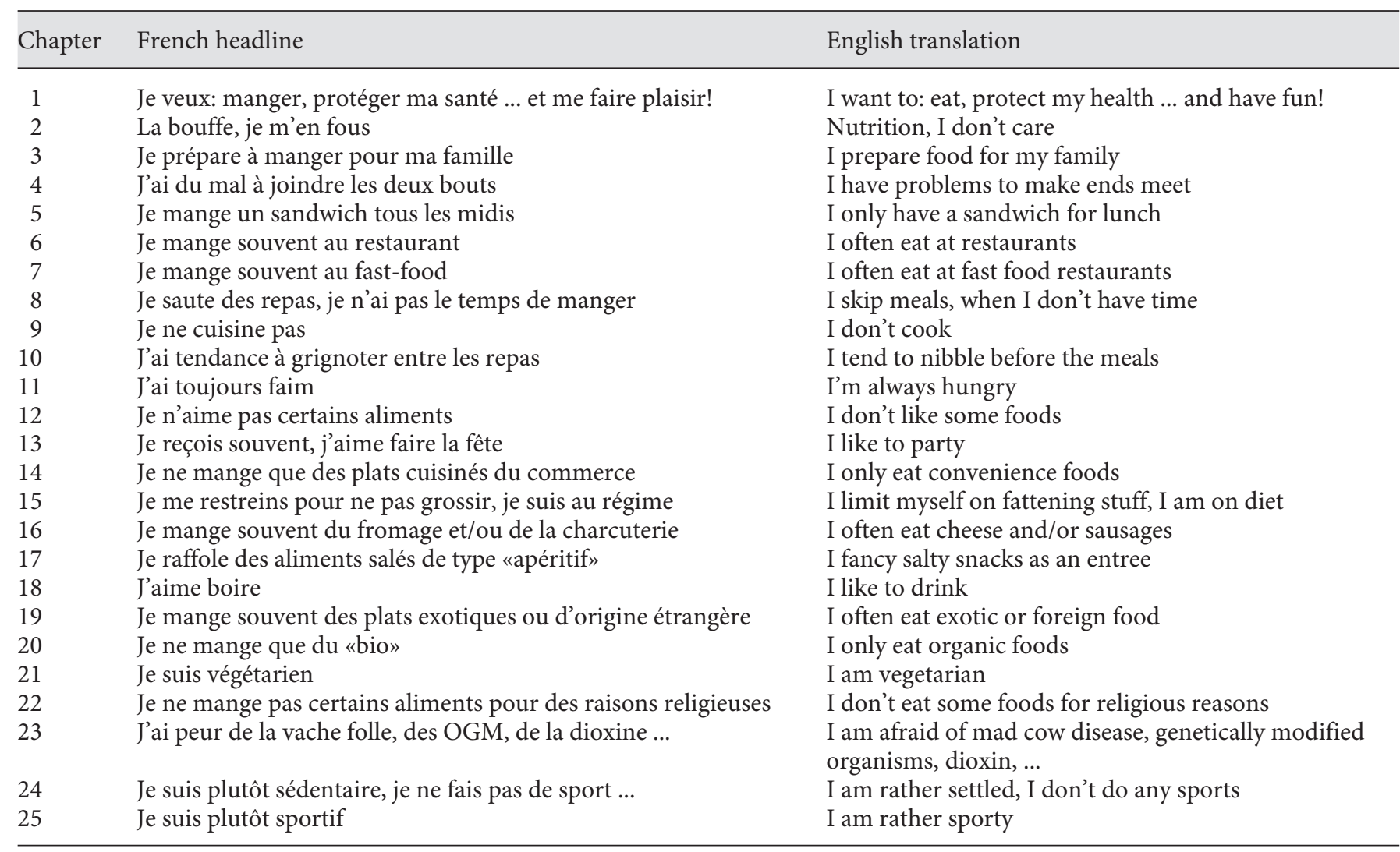

like quarter circle [13]. The red-colored inner section of the rainbow represents meat and alternatives, the adjacent blue section stands for milk products, the third green section for vegetables and fruits, and the outer section in yellow lists grain products as largest part of the rainbow. Together with pictures of the different foods of the four sections, the Canadian food guide states to enjoy a variety of foods from each group every day and to choose low-fat foods more often. Like other food guides, the Canadian food guide rainbow is supplemented by detailed recommendations on the amounts of servings and typical serving sizes for the four food groups. Grain products are recommended to be consumed from 5-12 servings per day, while 1 serving may be e.g. 1 slice of bread, $30 \mathrm{~g}$ of cold cereals, $175 \mathrm{ml}$ of hot cereals, etc. 5-10 servings of vegetables and fruits are suggested and to choose dark green and orange vegetables and orange fruit more often. In contrast to other examples of national FBDGs, the Canadian foodguide rainbow distinguishes its guideline on milk products by age groups: children of 4-9 years are recommended to daily consume $2-3$ servings, youth $10-16$ years and pregnant women should have 3-4 servings, and adults 2-
4 servings. It is also recommended to choose low-fat milk products more often. Similarly, low-fat meat and alternatives are recommended in form of leaner meats, poultry and fish, but also dried peas, beans and lentils.

In 2004, an extended version of the food-guide pyramid has been issued in form of the Canadian Guide to Healthy Eating and Physical Activity [14]. The quarter circle rainbow has been complemented to a half circle rainbow by adding recommendations on physical activity, advising to reduce sitting for long periods, to increase strength activities, to increase flexibility activities, and to increase endurance activities. Consequently, the Canadian Guide provides the most detailed and comprehensive recommendation on physical activity and puts this equal to the recommendations on nutrition by using the same area and format.

The FBDGs developed for the UK in form of the food plate has also been used as a role model for other countries (e.g. Australia) and can also be seen as the model used for the development of the German food circle. The original guideline shows the recommended food groups fruit and vegetables, bread cereals and potatoes, milk and 
dairy food, meat, fish and alternatives, and fatty and sugary foods according to their quantity recommended [15]. This visualization of the UK FBDGs comes together with eight worded guidelines, which are similar to those of other countries. These guidelines are:

- Base your meals on starchy foods

- Eat lots of fruit and vegetables

- Eat more fish

- Cut down on saturated fat and sugar

- Try to eat less salt - no more than $6 \mathrm{~g}$ a day

- Get active and try to be a healthy weight

- Drink plenty of water

- Don't skip breakfast.

The UK guidelines are a good example of the transferability of the concept to other countries and societies which are similar in cultural background and have similar nutrition issues to address. However, it is obvious that the process of adopting guidelines needs also to consider the particularities of the target population to gain the required acceptance in populations of different nutritional background.

\section{Concluding Remarks}

FBDGs are - if done properly - an elaborative task and require substantial amount of developmental work for many expert groups from various disciplines and responsibilities. They have to be regarded in the same way as any other marketing instrument to be successful. Enormous resources are therefore required not only for the develop- ment but also for the dissemination of the guidelines on a regular basis and with appropriate mass media coverage. In the presence of a wide range of food and nutrition information, whether brand- and product-specific or independent from industry interests, FBDGs need to stand out from the information overload in the nutrition sector.

It is mandatory in view of our globalized society that FBDGs are harmonized internationally, to avoid the intrusion of diverging guidelines from different countries. Obviously, the national or regional harmonization is crucial and should include information issued from governmental bodies, non-governmental organizations, and the private sector. FBDGs require constant development and adoption, to respond to a changing society, but this adoption has to proceed gently and wisely.

It is unfortunate that the nutrition community is inconsistent in its public appearance. Therefore, close networking involving all groups dealing with nutritional issues is vital to plan for clear and unbiased messages for the general public. Nutritional science, as any other natural science, is in a process of constant development and new findings may lead to changing evidence. However, this is a subtle process and sudden paradigm changes are actually rare to occur. Although it is difficult to disseminate information on a scientifically appropriate level with its amount of details required for scientific accuracy, it is important not to polarize the guidelines for the sake of comprehensibility. At the present state, it is obvious that FBDGs require a lot more resources to be successful and effective in improving nutrition of the target populations.

\section{References}

1 Food and Agriculture Organisation, World Health Organisation: International Conference on Nutrition. World Declaration on Nutrition and Plan of Action for Nutrition, Rome 1992.

2 World Health Organisation: Preparation and Use of Food-Based Dietary Guidelines. Report of a Joint FAO/WHO Consultation. WHO/NUT/96.6. Geneva, WHO, 1996.

3 Clay WD: Preparation and Use of FoodBased Dietary Guidelines. FNA/ANA 1997; 19:42-47.

4 United States Department of Agriculture: The Food Guide Pyramid. Washington, USDA, 1992.

5 Nishida C: WHO's Work on Development and Implementation of Food-Based Dietary Guidelines. EFSA Colloquium 5: Development of Food-Based Dietary Guidelines, Parma 2006.
6 Willett WC: Eat, Drink and Be Healthy. New York, Free Press, 2001.

7 United States Department of Agriculture: MyPyramid.gov - Steps to a Healthier You. http://www.mypyramid.gov (accessed 28.10.2006).

8 Food and Agriculture Organisation: National Dietary Guidelines. http://www.fao.org/ ag/agn/nutrition/education_guidelines_ en.stm (accessed 28.10.2006).

9 Ministry of Public Health: Manual Nutrition Flag. Healthy Eating for Thais. Working Group on Food-Based Dietary Guidelines For Thai People. Bangkok, Nutrition Division/Department of Health, 2001.
10 Schneeman BO: Preparation and Use of Food-Based Dietary Guidelines: Lessons from Thailand and the Philippines. FNA/ ANA 2001;28:55-64.

11 Ministry of Agriculture, Forestry and Fisheries of Japan: What Is 'Shokuiku (Food Education)'? Tokyo, MAFFJ, 2005.

12 Ministry of Health and Solidarity of France: La Santé Vient en Mangeant. Paris, MHSF, 2002.

13 Ministry of Public Works and Government Services Canada: Canada's Food Guide to Healthy Eating for People Four Years and Over. Ottawa, MPWGSC, 1997.

14 Government of Canada: Canadian Guide to Healthy Eating and Physical Activity. Ottawa, Government of Canada, 2004.

15 Department of Health of the United Kingdom: Food and Health Action Plan. London, DHUK, 2003. 